Pacific Journal of Mathematics

NOTE ON UNRESTRICTED REGULAR TRANSFORMATIONS 


\title{
A NOTE ON UNRESTRICTED REGULAR TRANSFORMATIONS
}

\author{
IV. R. UT Z
}

1. Introduction. Let $\mathbb{W}$ be the class of real continuous functions defined on the nonnegative reals and such that for each $g(t) \in \mathbb{W}^{\prime}$ the following conditions hold:

(a) $g(0)=0$ and $g(t)>0$ when $t>0$,

(b) for each triple $t_{1}, t_{2}, t_{3}, \geq 0$, the inequality $t_{1}+t_{2} \geq t_{3}$ implies $g\left(t_{1}\right)+$ $g\left(t_{2}\right) \geq g\left(t_{3}\right)$.

Let $M$ be a metric space wherein $[p, q]$ denotes the distance between $p, q \in M$. A transformation $T(M)=N$ is called unrestricted regular by W. Wilson [2] if there exists a $g(t) \in W$ such that for each pair $p, q \in W$ we have $[T(p), T(q)]$ $=g[p, q] \equiv g([p, q])$. The function $g$ (not always unique) is called a scale function for $T$.

It is easily seen that every member of the class $W$ is monotone increasing and that each unrestricted regular transformation is continuous and one-to-one. Thus an unrestricted regular transformation on a compact metric space is a homeomorphism. Wilson shows [2,p.65] that if $M$ is dense and metric and $T$ is unrestricted regular, then $T$ is a homeomorphism.

In $\$ 2$ of this note we examine the graphs of scale functions and show how the graph of the scale function of an unrestricted regular transformation determines the behavior of points under the transformation. Section 3 is devoted to a question involving a class of transformations investigated by E. J. Hickle [1] .

2. The graphs of scale functions. We shall establish the following result.

THEOREM 1 . If $M$ is a metric space and $T(M)=M$ is unrestricted regular with scale function $g(t)$, then for each $n=1,2,3, \cdots$, the transformation $T^{n}(M)=11$ is unrestricted regular with scale function $g^{n}(t)$ (that is, g iterated $n$ times).

Proof. Obviously $g^{n}(t)$ is real and continuous, $g^{n}(0)=0$, and $g^{n}(t)>0$ when $t>0$. Suppose $T^{n-1}(M)=M$ is unrestricted regular with scale function $g^{n-1}(t)$. 
Let $t_{1}+t_{2} \geq t_{3}$, where $t_{1}, t_{2}, t_{3} \geq 0$. Then

$$
g^{n-1}\left(t_{1}\right)+g^{n-1}\left(t_{2}\right) \geq g^{n-1}\left(t_{3}\right),
$$

and hence

$$
g^{n}\left(t_{1}\right)+g^{n}\left(t_{2}\right)=g\left[g^{n-1}\left(t_{1}\right)\right]+g\left[g^{n-1}\left(t_{2}\right)\right] \geq g\left[g^{n-1}\left(t_{3}\right)\right]=g^{n}\left(t_{3}\right) .
$$

Thus $g^{n}(t) \in W$. Also we have

$$
\begin{aligned}
{\left[T^{n}(p), T^{n}(q)\right]=[} & \left.T\left\{T^{n-1}(p)\right\}, T\left\{T^{n-1}(q)\right\}\right] \\
& =g\left[T^{n-1}(p), T^{n-1}(q)\right]=g\left(g^{n-1}[p, q]\right)=g{ }^{n}[p, q],
\end{aligned}
$$

for each pair $p, q \in M$. Thus, since $T$ is unrestricted regular with scale function $g(t)$, we have proved by induction that $T^{n}(M)=M$ is an unrestricted regular transformation with scale function $g^{n}(t)$.

If $M$ is a metric space of at least two points, $p \in M$, and $T(M)=M$ is unrestricted regular, then we shall call the set $\sum_{n=0}^{\infty} T^{n}(p) \subset M$ the orbit of $p$ under $T$.

Let $g(t)$ be a scale function for $T$. We distinguish three cases.

CASE I. If $g(t)<t$ for all $t>0$, then each pair of points of $M$ will determine asymptotic orbits. That is, given $p, q \in M$ and $\epsilon>0$, there exists an integer $N$ such that $\left[T^{n}(p), T^{n}(q)\right]<\epsilon$ for all $n>N$.

Proof. Let $p$ and $q$ be points of $M$. Since $g(t)<t$, we see that $\left[T^{n}(p), T^{n}(q)\right]$ $=g^{n}[p, q]$ decreases monotonically as $n$ increases. Suppose that the monotone decreasing sequence of real numbers $[p, q], g[p, q], g^{2}[p, q], \cdots$, has $u \neq 0$ as limit point. Choose $\delta$ such that $0<\delta<u$, and let $s$ be the greatest lower bound of $t-g(t)$ on the interval $u-\delta \leq t \leq u+\delta$. Since $u$ is the limit point of the sequence, there exists an integer $n$ for which $g^{n}[p, q]-u<\min (s, \delta)$. Since $g^{n}[p, q]$ is in the interval $u-\delta \leq t \leq u+\delta$, it follows that $g^{n}[p, q]-g^{n+1}[p, q]$ $\geq s$ and $u-g^{n+1}[p, q]>0$. Thus for all $i>n$, the elements $g^{i}[p, q]$ of the sequence are smaller than $u$; this contradicts the assumption that $u \neq 0$ is the limit point of the sequence.

In Case I, $T$ has equicontinuous powers.

CASE II. If $g(t)>t$ for all $t>0$, then $T$ is unstable. That is, there exists a $\delta>0$ (in this case any positive number will serve) such that if $p, q \in M$, then there is an integer $N$ for which $n>N$ implies $\left[T^{n}(p), T^{n}(q)\right]>\delta$. 
CASE III. (1) If $g(t) \equiv t$, then all orbits are parallel. That is, $T$ is an isometry. If $g(t) \not \equiv t$, there are these possibilities:

(2a) When $g[p, q]=[p, q]$, the orbits of $p$ and $q$ are parallel (as in Case III).

(2b) If $g[p, q]>[p, q]$, and if there is a zero of $g(t)-t$ greater than $[p, q]$, then the orbits of $p$ and $q$ approach a distance apart equal to the first zero of $g(t)-t$ that is greater than $[p, q]$. If no zero of $g(t)-t$ is greater than $[p, q]$, the orbits of $p$ and $q$ separate as in Case II.

(2c) If $g[p, q]<[p, q]$, and if no positive zero of $g(t)-t$ is smaller than $[p, q]$, then $p$ and $g$ have asymptotic orbits as in Case I. If $g(t)-t$ has a positive zero smaller than $[p, q]$, then the orbits of $p$ and $q$ approach a distance apart equal to the first zero of $g(t)-t$ less than $[p, q]$.

The proofs of these cases are similar to the proof of Case I.

Theorem 2. If $M$ is a bounded metric space, then Case I and Case II are not possible.

Proof. That Case II cannot occur is obvious.

Suppose $g(t)<t$ (Case I). Let $\delta$ be the least upper bound of $[p, q]$ for all $p, q \in$ i. Let $\sigma>0$ be the greatest lower bound for $t-g(t)$ on the interval $\delta / 2 \leq t \leq \delta$. Select $p, q \in M$ such that $[p, q]>\max (\delta-\sigma, \delta / 2)$. Since $T^{-1}(p), T^{-1}(q)$ are elements of $M$, and since

$$
\left[T^{-1}(p), T^{-1}(q)\right]>g\left[T^{-1}(p), T^{-1}(q)\right]=[p, q],
$$

it follows that

$$
(1 / 2) \delta \leq\left[T^{-1}(p), T^{-1}(q)\right] \leq \delta
$$

Thus,

$$
[p, q]=g\left[T^{-1}(p), T^{-1}(q)\right]<\left[T^{-1}(p), T^{-1}(q)\right]-\sigma \leq \delta-\sigma ;
$$

this contradicts $[p, q]>\delta-\sigma$ and completes the proof of the theorem.

Lemma 1 . If $g(t) \in W$, then there exists a real number s such that, on $0<t$ $\leq s$, either (i) $g(t) \equiv t$, or (ii) $g(t)>t$, or (iii) $g(t)<t$.

Proof. Suppose that $g(t) \not \equiv t$ on every interval $0<t \leq s$. If $t=0$ is not a limit point of the positive zeros of $g(t)-t$, then obviously on some interval $0<t \leq s$ we have $g(t)<t$ or $g(t)>t$. Suppose that $t=0$ is a limit point of the zeros of 
$g(t)-t$ and suppose that in every interval $0<t \leq s$ there are values of $t$ for which $g(t)<t$ and $g(t)>t$. Select $u_{1}$ and $u_{2}$ such that $g\left(u_{1}\right)=u_{1}$ and $g\left(u_{2}\right)=u_{2}$. and such that $g(t)>t$ on the interval $u_{1}<t<u_{2}$. Select $u_{3}>0$ such that $g\left(u_{3}\right)$ $<u_{3}$ and $u_{3}<u_{2}-u_{1}$. Define $u_{4}=u_{1}+u_{3}$. Since $u_{1}<u_{4}<u_{2}$, we have $g\left(u_{4}\right)$ $>u_{4}$. Since $u_{1}+u_{3} \geq u_{4}$, we must have $g\left(u_{1}\right)+g\left(u_{3}\right) \geq g\left(u_{4}\right)$. This is not the case since $g\left(u_{1}\right)+g\left(u_{3}\right)=u_{1}+g\left(u_{3}\right)<u_{1}+u_{3}=u_{4}<g\left(u_{4}\right)$. Thus on some interval $0<t \leq s$, either $g(t) \leq t$ or $g(t) \geq t$.

We must now eliminate the possibility of the equalities. Suppose $g(t) \leq t$ on $0<t \leq s$ but there is no subinterval $0<t \leq s_{1}$ on which $g(t)<t$ or $g(t) \equiv t$. Let $u \leq s$ be such that $g(u)=u$. Select $v<u$ such that $g(v)<v$. Now, $v+$ $(u-v)=u$; but

$$
g(v)+g(u-v) \leq g(v)+(u-v)<v+u-v=u=g(u),
$$

and property $(b)$ of $g(t)$ is violated. Thus $g(t)<t$.

If $g(t) \geq t$ on $0<t \leq s$, but there is no subinterval $0<t \leq s_{1}$ on which $g(t)$ $>t$ or $g(t) \equiv t$, then choose $0<u_{1}<s$ and $0<u_{2} \leq s$ such that $g\left(u_{1}\right)=u_{1}$ and $g\left(u_{2}\right)=u_{2}$, and such that on the $t$-interval $0<u_{1}<t<u_{2} \leq s$ we have $g(t)>t$. Select $0<u_{3}<u_{2}-u_{1}$ such that $g\left(u_{3}\right)=u_{3}$ and define $u_{4}=u_{3}+u_{1}$. Then

$$
g\left(u_{3}\right)+g\left(u_{1}\right)=u_{3}+u_{1}=u_{4}<g\left(u_{4}\right),
$$

since $u_{1}<u_{4}<u_{2}$. Thus $g(t)$ fails to have property $(b)$. We conclude that $g(t)>t$. This proves the lemma.

LEMMA 2. If (i) of Lemma 1 occurs, then either $g(t)=t$ for all $t>0$ or there exists an $r>0$ such that $g(t)=t$ for $0 \leq t \leq r$ and $g(t)<t$ for all $t>r$. If (iii) of Lemma 1 occurs, then $g(t)<t$ for all $t>0$.

Proof. Suppose that (i) of Lemma 1 occurs. Let $r$ be the largest value of $s$ for which $g(t)=t$ on $0 \leq t \leq s$ (if $r$ does not exist, then $g(t)=t$ for all $t>0$ ). Let $t$ be any real number greater than $r$. Suppose $g(t)>t$. Then $t=m r+q$, where $m$ is a positive integer and $0 \leq q<r$. Since $g(r)=r$, we have $g(m r) \leq m g(r)=m r$; and since $0 \leq q<r$, we have $g(q)=q$. Hence

$$
g(m r)+g(q) \leq m r+q=t<g(t),
$$

in violation of property $(b)$ of $g(t)$. Thus $g(t) \leq t$ for all $t>0$. Suppose $t>r$ and $g(t)=t$. Then there exists a nonnegative integer $m$, and real numbers $u$ and $q$ such that $m r+q+u=t$, and such that $g(u)<u$. However, 


$$
g(m r)+g(q)+g(u) \leq m r+q+g(u)<m r+q+u=t=g(t),
$$

and condition $(b)$ of $g(t)$ is violated. Thus $g(t)<t$ for $t>r$, and the first part of the lemma is proved.

Suppose that (iii) of Lemma 1 occurs. To show that $g(t)<t$ for all real values of $t$, we shall show that for no $t>0$ is $g(t)=t$. If $g(t)=t$ for some $t>0$, then there exists a smallest value $u$ of $t$ such that $g(u)=u$. Now, $g(u / 2)<u / 2$ since $u$ is the smallest value of $t$ for which $g(t)=t$. Hence $g(u / 2)+g(u / 2)<u$, contrary to property $(b)$ of $g(t)$. This completes the proof of the lemma.

THEOREM 3. If $M$ is a bounded metric space and $T(M)=M$ is unrestricted regular and has equicontinuous powers, then $T$ is an isometry.

Proof. Since $T$ has equicontinuous powers, given $\epsilon>0$ there exists $\delta>0$ such that when $[p, q]<\delta$ we have $\left[T^{n}(p), T^{n}(q)\right]<\epsilon$ for $n=1,2,3, \cdots$. From this it follows that (ii) of Lemma 1 cannot occur. For if $\epsilon$ is taken as $s / 2$ in Lemma 1 , then regardless of the size of $[p, q]$, we have $\left[T^{n}(p), T^{n}(q)\right]>s / 2$ for $n$ sufficiently large (cf. $2 b$ of Case III).

Further, (iii) of Lemma 1 cannot occur since by Lemma 2 this implies Case I, which is impossible since ith is bounded.

Since (i) of Lemma 1 must occur, either $g(t) \equiv t$ for $t>0$, or there exists an $r>0$ such that $g(t)=t$ for all $0 \leq t \leq r$ and $g(t)<t$ for all $t>r$. If $g(t) \not \equiv t$, then we can show by the argument of Theorem 2 that distances in $M$ are bounded by $r$. Hence we always have $[T(p), T(q)]=g[p, q]$ for each pair $p, q \in h$, and $T$ is an isometry.

REMARK. Suppose that (ii) of Lemma 1 occurs and suppose that $g(t)-t$ has a positive zero. We can show easily that either there exist arbitrarily large zeros of $g(t)-t$ or there exists a real number $w>0$ such that $t>w$ implies $g(t)<t$. If $r$ is the smallest positive zero of $g(t)-t$, and $N$ is the length of any interval of the $t$-axis on which $g(t)>t$, then $N \leq r$.

The following theorem relates periodicity to unrestricted regularity. Other theorems of this nature are possible.

THEOREM 4. Let $M$ be a metric space. If $T(M)=M$ is pointwise periodic and unrestricted regular then $T$ is an isometry.

Proof. Let $p$ and $q$ be arbitrary points of $M$. Since $p$ and $q$ are individually 
periodic (possibly having different periods), there exists an integer $n$ (in particular, the products of the periods of $p$ and $q$ will serve) such that $T^{n}(p)=p$ and $T^{n}(q)=q$. Thus $p$ and $q$ are fixed under $T^{n}$. If $g(t)$ is the scale function of $T$, then $g^{n}(t)$ is the scale function of $T^{n}$. Since $p$ and $q$ are fixed under $T^{n}$, we have

$$
\mathrm{g}^{n}\left[T^{n}(p), T^{n}(q)\right]=\left[T^{n}(p), T^{n}(q)\right]=[p, q] .
$$

Thus we have $g^{n}[p, q]=[p, q]$. This implies that $g[p, q]=[p, q]$; and since $g$ is the scale function for $T$, we have $[T(p), T(q)]=g[p, q]=[p, q]$, and the theorem is proved.

3. A class of transformations. Given a metric space $M$, Mickle [1] defines the associated class $P(M)$ of real continuous functions on the nonnegative reals as those functions $g(t)$ satisfying these conditions:

(a) $g(0)=0$ and $g(t)>0$ when $t>0$,

(b) for any $m+1$ points $p_{0}, p_{1}, p_{2}, \cdots, p_{m}$ in $M$ the real quadratic form

$$
\sum_{i, j=1}^{m}\left\{g\left[p_{0}, p_{i}\right]^{2}+g\left[p_{0}, p_{J}\right]^{2}-g\left[p_{i}, p_{j}\right]^{2}\right\} \xi_{i} \xi_{j}
$$

is positive definite.

For example, let $U$ be any set with metric $[p, q]=1$ for $p \neq q,[p, q]=0$ for $p=q$. Let $g(t)$ be any real continuous function that satisfies condition $(a)$. If $p_{0}, p_{1}, \cdots, p_{m}$ are any set of $m+1$ distinct points of $M$, then $g\left[p_{i}, p_{j}\right]=g(1)$ $=a>0$ for $i \neq j$. The elements of the matrix $\left\|a_{i, j}\right\|$ of the quadratic form of condition (b) are $2 a^{2}$ if $i=j$ and $a^{2}$ if $i \neq j$. From this, and from well-known theorems concerning quadratic forms, it follows that condition $(b)$ is always satisfied. lience, in this case, $P(M)$ consists of all real continuous functions for which $(a)$ holds.

Let $T(M)=N$ be a continuous transformation. Then $T$ is said by Mickle to satisfy the condition $C(g), g(t) \in P(M)$, if for each pair $p, q \in M$ we have $[T(p), T(q)] \leq g[p, q]$.

A transformation may satisfy the condition $C(g)$ for some $g(t) \in P(M)$, yet not be unrestricted regular. Let $M$ be the interval $0 \leq x \leq 1$ with the metric described in the second paragraph of this section. Let $N$ be the same interval 
with the Euclidean metric. Let $T(M)=N$ be the identity on the point set. That is, if $p \in M$ has coordinate $x$, then $T(p) \in N$ has coordinate $x$. If $g(t) \in P(M)$ and $g(1) \geq 1$, then for each distinct pair $p, q \in M$, we have $[T(p), T(q)] \leq 1 \leq$ $g[p, q]$, and $T$ satisfies $C(g)$. However, $T$ is not unrestricted regular.

QUESTION. Suppose that $T(M)=N$ is an unrestricted regular transformation. When does there exist an element $g(t) \in P(M)$ such that $T$ satisfies the condition $C(g)$ ?

\section{REFERENCES}

1. E. J. Mickle, On the extension of a transformation, Bull. Amer. Math. Soc. 55 (1949), 160-164.

2. W. A. Wilson, On certain types of continuous transformations of metric spaces, Amer. J. Math. 57 (1935), 62-68.

UNIVERSITY OF MisSOURI 



\title{
PACIFIC JOURNAL OF MATHEMATICS
}

\section{EDITORS}

\author{
HERBERT BUSEMANN \\ University of Southern California \\ R. M. RoBINSON \\ Los Angeles 7, California \\ University of California \\ Berkeley 4, California \\ E. F. BECKENBACH, Managing Editor \\ University of California \\ Los Angeles 24, California
}

\section{ASSOCIATE EDITORS}

R. P. DILWORTH
HERBERT FEDERER
MARSHALL HALL

\author{
P. R. HALMOS \\ HEINZ HOPF \\ R. D. JAMES
}

\author{
B $\emptyset$ RGE JESSEN \\ PAUL LÉVY \\ GEORGE PÓLYA
}

\author{
J. J. STOKER \\ E. G. STRAUS \\ KÔSAKU YOSIDA
}

\section{SPONSORS}

UNIVERSITY OF BRITISH COLUMBIA

CALIFORNIA INSTITUTE OF TECHNOLOGY

UNIVERSITY OF CALIFORNIA, BERKELEY

UNIVERSITY OF CALIFORNIA, DAVIS

UNIVERSITY OF CALIFORNIA, LOS ANGELES

UNIVERSITY OF CALIFORNIA, SANTA BARBARA

OREGON STATE COLLEGE

UNIVERSITY OF OREGON

\author{
UNIVERSITY OF SOUTHERN CALIFORNIA \\ STANFORD UNIVERSITY \\ WASHINGTON STATE COLLEGE \\ UNIVERSITY OF WASHINGTON

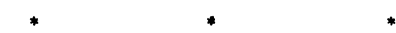 \\ AMERICAN MATHEMATICAL SOCIETY \\ NATIONAL BUREAU OF STANDARDS, \\ INSTITUTE FOR NUMERICAL ANALYSIS
}

Mathematical papers intended for publication in the Pacific Journal of Mathematics should be typewritten (double spaced), and the author should keep a complete copy. Manuscripts may be sent to any of the editors. All other communications to the editors should be addressed to the managing editor, E. F. Beckenbach, at the address given above.

Authors are entitled to receive 100 free reprints of their published papers and may obtain additional copies at cost.

The Pacific Journal of Mathematics is published quarterly, in March, June, September, and December. The price per volume (4 numbers) is $\$ 8.00$; single issues, $\$ 2.50$. Spécial price to individual faculty members of supporting institutions and to members of the American Mathematical Society: $\$ 4.00$ per volume; single issues, $\$ 1.25$.

Subscriptions, orders for back numbers, and changes of address should be sent to the publishers, University of California Press, Berkeley 4, California.

UNIVERSTTY OF CALIFORNIA PRESS • BERKELEY AND LOS ANGELES 


\section{Pacific Journal of Mathematics}

\section{Vol. 1, No. $3 \quad$ BadMonth, 1951}

R. P. Boas, Completeness of sets of translated cosines ............. 321

J. L. Brenner, Matrices of quaternions . ..................... 329

Edmond Darrell Cashwell, The asymptotic solutions of an ordinary differential equation in which the coefficient of the parameter is

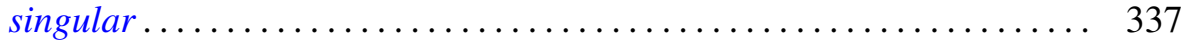

James Dugundji, An extension of Tietze's theorem ................ 353

John G. Herriot, The polarization of a lens ...................... 369

J. D. Hill, The Borel property of summability methods ............... 399

G. G. Lorentz, On the theory of spaces $\Lambda \ldots \ldots \ldots \ldots \ldots \ldots \ldots \ldots \ldots . \ldots \ldots$

J. H. Roberts and W. R. Mann, On a certain nonlinear integral equation of the Volterra type ................................. 431

W. R. Utz, A note on unrestricted regular transformations . . .......... 447

Stanley Simon Walters, Remarks on the space $H^{p} \ldots \ldots \ldots \ldots \ldots \ldots . . \ldots 55$

Hsien Chung Wang, Two theorems on metric spaces ............... 473 